\title{
Linearization of Local Cohomology Modules
}

\author{
Josep Àlvarez Montaner and Santiago Zarzuela
}

\begin{abstract}
Let $k$ be a field of characteristic zero and $R=k\left[x_{1}, \ldots, x_{n}\right]$ the polynomial ring in $n$ variables. For any ideal $I \subset R$, the local cohomolgy modules $H_{I}^{i}(R)$ are known to be regular holonomic $A_{n}(k)$-modules. If $k$ is the field of complex numbers, by the Riemann-Hilbert correspondence there is an equivalence of categories between the category of regular holonomic $\mathcal{D}_{X^{-}}$ modules and the category Perv $\left(\mathbb{C}^{n}\right)$ of perverse sheaves. Let $T$ be the union of the coordinate hyperplanes in $\mathbb{C}^{n}$, endowed with the stratification given by the intersections of its irreducible components and denote by $\operatorname{Perv}^{T}\left(\mathbb{C}^{n}\right)$ the subcategory of Perv $\left(\mathbb{C}^{n}\right)$ of complexes of sheaves of finitely dimensional vector spaces on $\mathbb{C}^{n}$ which are perverse relatively to the given stratification of $T$. This category has been described in terms of linear algebra by Galligo, Granger and Maisonobe. If $M$ is a local cohomology module $H_{I}^{i}(R)$ supported on a monomial ideal, one can see that the equivalent perverse sheaf belongs to Perv ${ }^{T}\left(\mathbb{C}^{n}\right)$. Our main purpose in this note is to give an explicit description of the corresponding linear structure, in terms of the natural $\mathbb{Z}^{n}$-graded structure of $H_{I}^{i}(R)$. One can also give a topological interpretation of this linear structure, recovering as a consequence the results on the structure of local cohomology modules supported on squarefree monomial ideals given by M. Mustaţă.
\end{abstract}

\section{Introduction}

Let $k$ be a field of characteristic zero and $R=k\left[x_{1}, \ldots, x_{n}\right]$ the polynomial ring in $n$ variables. For any ideal $I \subset R$, the local cohomolgy modules $H_{I}^{i}(R)$ are known since long to have a module structure over the Weyl algebra $A_{n}(k)$, more precisely, they are regular holonomic $A_{n}(k)$-modules. This structure has been fruitfully used in recent years to prove interesting properties of the local cohomolgy modules $H_{I}^{i}(R)$, as it was first realized by G. Lyubeznik [9], and later by several other authors (see e.g. [1], [2], [11] and [12]), particularly in the case of monomial ideals.

Assume now that $k$ is the field of complex numbers and let $X=\mathbb{C}^{n}$, let $\mathcal{O}_{X}$ be the sheaf of holomorphic functions in $\mathbb{C}^{n}$, and let $\mathcal{D}_{X}$ be the sheaf of differential

1991 Mathematics Subject Classification. Primary 13D45; Secondary 32C38.

Key words and phrases. Local cohomology, monomial ideals, $\mathcal{D}$-modules.

The first author was partially supported by the University of Nice.

The second author has been partially supported by DGCYT BFM2001-3584. 
operators in $\mathbb{C}^{n}$ with holomorphic coefficients. By the Riemann-Hilbert correspondence there is an equivalence of categories between the category of regular holonomic $\mathcal{D}_{X}$-modules and the category Perv $\left(\mathbb{C}^{n}\right)$ of perverse sheaves. Let $T$ be the union of the coordinate hyperplanes in $\mathbb{C}^{n}$, endowed with the stratification given by the intersections of its irreducible components. Denote by Perv ${ }^{T}\left(\mathbb{C}^{n}\right)$ the subcategory of Perv $\left(\mathbb{C}^{n}\right)$ of complexes of sheaves of finitely dimensional vector spaces on $\mathbb{C}^{n}$ which are perverse relatively to the given stratification of $T$ ([6, I.1], and by $\operatorname{Mod}\left(\mathcal{D}_{X}\right)_{h r}^{T}$ the full abelian subcategory of the category of regular holonomic $\mathcal{D}_{X}$-modules such that their solution complex $\mathbb{R} \operatorname{Hom}_{\mathcal{D}_{X}}\left(\mathcal{M}, \mathcal{O}_{X}\right)$ is an object of $\operatorname{Perv}^{T}\left(\mathbb{C}^{n}\right)$. Then, the above equivalence gives by restriction an equivalence of categories between $\operatorname{Mod}\left(\mathcal{D}_{X}\right)_{h r}^{T}$ and $\operatorname{Perv}{ }^{T}\left(\mathbb{C}^{n}\right)$. Moreover, this last category has been described in terms of linear algebra by Galligo, Granger and Maisonobe in [6].

Let $M$ be a $A_{n}(\mathbb{C})$-module. Then, $\mathcal{M}^{a n}=\mathcal{O}_{X} \otimes_{R} M$ has a natural structure of $\mathcal{D}_{X}$-module. In this way, regular holonomic $A_{n}(\mathbb{C})$-modules may be considered as regular holonomic $\mathcal{D}_{X}$-modules and, for those such that $\mathcal{M}^{a n} \in \operatorname{Mod}\left(\mathcal{D}_{X}\right)_{h r}^{T}$ (e.g. local cohomology modules supported on monomial ideals), one can describe the linear structure of the corresponding perverse sheaf in terms of the module $M$ itself, see [7]. Our main purpose in this note is to give an explicit description of this linear structure when $M$ is a local cohomology module supported on a monomial ideal. It will be expressed in terms of the natural $\mathbb{Z}^{n}$-graded structure of these local cohomology modules, coming from the polynomial ring $\mathbb{C}\left[x_{1}, \ldots, x_{n}\right]$ by giving $\operatorname{deg} x_{i}=\varepsilon_{i}$, where $\varepsilon_{1}, \ldots, \varepsilon_{n}$ denotes the canonical basis of $\mathbb{Z}^{n}$.

More generally, we will consider the category of straight modules introduced by $\mathrm{K}$. Yanagawa [13]. For any field $k$, it is a full abelian subcategory of the category of $\mathbb{Z}^{n}$-graded $R$-modules, which includes the local cohomology modules $H_{I}^{i}(R)$ supported on a monomial ideal $I$. For $k=\mathbb{C}$, it is proven in [2] that a slight variation of this category (the category of $\varepsilon$-straight modules) is equivalent to the full abelian subcategory of $\operatorname{Mod}\left(\mathcal{D}_{X}\right)_{h r}^{T}$ whose equivalent perverse sheaves have variation zero. The proof of this result is based on the fact that the simple objects in both categories coincide, and that the objects in each category admit similar finite increasing filtrations, such that the quotients are a finite direct sum of simple objets.

Here, for such a $\operatorname{Mod}\left(\mathcal{D}_{X}\right)_{h r}^{T}$-module we want to make precise the linear description of its equivalent perverse sheaf in terms of the corresponding $\varepsilon$-straight module. Moreover, in the case of the local cohomology modules supported on squarefree monomial ideals, we shall give a topological interpretation of this linear description, recovering, as a consequence, the results on the structure of local cohomology modules supported on squarefree monomial ideals given by M. Mustață $[10]$.

For any unexplained terminology concerning the theory of $\mathcal{D}$-modules we shall use [3], [4] or [5].

\section{Preliminaries}

As said in the introduction, for any regular holonomic module $\mathcal{D}_{X}$-module in $\operatorname{Mod}\left(\mathcal{D}_{X}\right)_{h r}^{T}$ its solution complex $\mathbb{R} \operatorname{Hom}_{\mathcal{D}_{X}}\left(\mathcal{M}, \mathcal{O}_{X}\right)$ is an object of $\operatorname{Perv}^{T}\left(\mathbb{C}^{n}\right)$, 
and by the Riemann-Hilbert correspondence the functor of solutions establishes an equivalence of categories between $\operatorname{Mod}\left(\mathcal{D}_{X}\right)_{h r}^{T}$ and $\operatorname{Perv}^{T}\left(\mathbb{C}^{n}\right)$.

In [6], the category $\operatorname{Perv}^{T}\left(\mathbb{C}^{n}\right)$ has been linearized as follows: Let $\mathcal{C}_{n}$ be the category whose objects are families $\left\{\mathcal{M}_{\alpha}\right\}_{\alpha \in\{0,1\}^{n}}$ of finitely dimensional complex vector spaces, endowed with linear maps

$$
\mathcal{M}_{\alpha} \stackrel{u_{i}}{\longrightarrow} \mathcal{M}_{\alpha+\varepsilon_{i}}, \mathcal{M}_{\alpha} \stackrel{v_{i}}{\longleftarrow} \mathcal{M}_{\alpha+\varepsilon_{i}}
$$

for each $\alpha \in\{0,1\}^{n}$ such that $\alpha_{i}=0$. These maps are called canonical (resp., variation) maps, and they are required to satisfy the conditions:

$$
u_{i} u_{j}=u_{j} u_{i}, \quad v_{i} v_{j}=v_{j} v_{i}, \quad u_{i} v_{j}=v_{j} u_{i} \text { and } v_{i} u_{i}+i d \text { is invertible. }
$$

Such an object will be called an $n$-hypercube. A morphism between two $n$-hypercubes $\left\{\mathcal{M}_{\alpha}\right\}_{\alpha}$ and $\left\{\mathcal{N}_{\alpha}\right\}_{\alpha}$ is a set of linear maps $\left\{f_{\alpha}: \mathcal{M}_{\alpha} \rightarrow \mathcal{N}_{\alpha}\right\}_{\alpha}$, commuting with the canonical and variation maps (see [7]). It is proved in [loc.cit.] that there is an equivalence of categories between $\operatorname{Perv}{ }^{T}\left(\mathbb{C}^{n}\right)$ and $\mathcal{C}_{n}$.

We have to point out that the functor $\operatorname{Mod}\left(\mathcal{D}_{X}\right)_{h r}^{T} \longrightarrow \mathcal{C}_{n}$ is a contravariant exact functor and, given an object $\mathcal{M}$ of $\operatorname{Mod}\left(\mathcal{D}_{X}\right)_{h r}^{T}$, its corresponding $n$-hypercube is constructed as follows:

Consider $\mathbb{C}^{n}=\prod_{i=1}^{n} \mathbb{C}_{i}$, with $\mathbb{C}_{i}=\mathbb{C}$ for $1 \leq i \leq n$, let $K_{i}=\mathbb{R}^{+} \subset \mathbb{C}_{i}$ and set $V_{i}=\mathbb{C}_{i} \backslash K_{i}$. For any $\alpha=\left(\alpha_{1}, \ldots, \alpha_{n}\right) \in\{0,1\}^{n}$ denote

$$
\mathcal{S}_{\alpha}:=\frac{\Gamma_{\prod_{i=1}^{n} V_{i}} \mathcal{O}_{X}}{\sum_{\alpha_{k}=1} \Gamma_{\mathbb{C}_{k} \times \prod_{i \neq k} V_{i} \mathcal{O}_{X}}} .
$$

Denoting with a subscript 0 the stalk at the origin, one has:

i) The vertices of the $n$-hypercube associated to $\mathcal{M}$ are the vector spaces $\mathcal{M}_{\alpha}:=\operatorname{Hom}_{\mathcal{D}_{0}}\left(\mathcal{M}_{0}, S_{\alpha, 0}\right)$.

ii) The linear maps $u_{i}$ are those induced by the natural quotient maps $\mathcal{S}_{\alpha} \rightarrow$ $\mathcal{S}_{\alpha+\varepsilon_{i}}$.

iii) The linear maps $v_{i}$ are the partial variation maps around the coordinate hyperplanes, i.e. for any $\varphi \in \operatorname{Hom}_{\mathcal{D}_{0}}\left(\mathcal{M}_{0}, \mathcal{S}_{\alpha, 0}\right)$ one has $\left(v_{i} \circ u_{i}\right)(\varphi)=$ $\Phi_{i}(\varphi)-\varphi$, where $\Phi_{i}$ is the partial monodromy around the hyperplane $x_{i}=0$.

The following is proved as well in [7]:

iv) If $C C(\mathcal{M})=\sum m_{\alpha} T_{X_{\alpha}}^{*} \mathbb{C}^{n}$ is the characteristic cycle of $\mathcal{M}$, then for all $\alpha \in\{0,1\}^{n}$ one has the equality $\operatorname{dim}_{\mathbb{C}} \mathcal{M}_{\alpha}=m_{\alpha}$.

Remark 2.1. Since $\mathcal{M}_{0}$ is a regular holonomic $\mathcal{D}_{X, 0}$-module in order to determine the solutions $\operatorname{Hom}_{\mathcal{D}_{X, 0}}\left(\mathcal{M}_{0}, \mathcal{S}_{\alpha, 0}\right)$ we only have to consider those Nilsson 
class functions in $\mathcal{S}_{\alpha, 0}$, i.e. those finite sums

$$
f=\sum_{\beta, m} \varphi_{\beta, m}(\mathbf{x})(\log \mathbf{x})^{m} \mathbf{x}^{\beta}
$$

where $\varphi_{\beta, m}(\mathbf{x}) \in \mathbb{C}\{\mathbf{x}\}, \beta \in \mathbb{C}$ and $m \in\left(\mathbb{Z}^{+}\right)^{n}$ contained in $S_{\alpha, 0}$ (see [3] for details). So, if $\mathcal{N}$ denotes the set of Nilsson class functions then:

$$
\mathcal{M}_{\alpha}:=\operatorname{Hom}_{\mathcal{D}_{X, 0}}\left(\mathcal{M}_{0}, \mathcal{N}_{\alpha}\right) \text {, where } \mathcal{N}_{\alpha}=\mathcal{N} \cap \mathcal{S}_{\alpha, 0} .
$$

\section{3. $\mathcal{D}_{X}$-modules with variation zero}

Among the objects of the category $\operatorname{Mod}\left(\mathcal{D}_{X}\right)_{h r}^{T}$ we will be interested in those having the following property:

Definition 3.1. We say that an object $\mathcal{M}$ of $\operatorname{Mod}\left(\mathcal{D}_{X}\right)_{h r}^{T}$ has variation zero if the morphisms $v_{i}: \operatorname{Hom}_{\mathcal{D}_{X, 0}}\left(\mathcal{M}_{0}, \mathcal{S}_{\alpha+\varepsilon_{i}, 0}\right) \longrightarrow \operatorname{Hom}_{\mathcal{D}_{X, 0}}\left(\mathcal{M}_{0}, \mathcal{S}_{\alpha, 0}\right)$ are zero for all $1 \leq i \leq n$ and all $\alpha \in\{0,1\}^{n}$ with $\alpha_{i}=0$.

Modules with variation zero form a full abelian subcategory of $\operatorname{Mod}\left(\mathcal{D}_{X}\right)_{h r}^{T}$ that will be denoted $\mathcal{D}_{v=0}^{T}$. In the sequel, we will denote by $\mathcal{C}_{n, v=0}$ the corresponding full abelian subcategory of $\mathcal{C}_{n}$ of $n$-hypercubes having variation zero.

In [2] it is proven that a slight variation of the category of straight modules introduced by $\mathrm{K}$. Yanagawa [13] is equivalent to the category $\mathcal{D}_{v=0}^{T}$ of modules with variation zero. More precisely, let $R=\mathbb{C}\left[x_{1}, \ldots, x_{n}\right]$ be the polynomial ring with coefficients in $\mathbb{C}$ and $A_{n}(\mathbb{C})$ the corresponding Weyl algebra.

Let $M$ be a graded $R$-module and $\alpha \in \mathbb{Z}^{n}$. As usual, we denote by $M(\alpha)$ the graded $R$-module whose underlying $R$-module structure is the same as that of $M$ and where the grading is given by $(M(\alpha))_{\beta}=M_{\alpha+\beta}$. If $\alpha \in \mathbb{Z}^{n}$, we set $\operatorname{supp}_{+}(\alpha)=\left\{i \mid \alpha_{i}>0\right\}$. We recall now the following definition of K. Yanagawa:

Definition 3.2. $([\mathbf{1 3}, 2.7])$ A $\mathbb{Z}^{n}$-graded module $M$ is said to be straight if the following two conditions are satisfied:

i) $\operatorname{dim}_{k} M_{\alpha}<\infty$ for all $\alpha \in \mathbb{Z}^{n}$.

ii) The multiplication map $M_{\alpha} \ni y \mapsto \mathbf{x}^{\beta} y \in M_{\alpha+\beta}$ is bijective for all $\alpha, \beta \in \mathbb{Z}^{n}$ with $\operatorname{supp}_{+}(\alpha+\beta)=\operatorname{supp}_{+}(\alpha)$.

The full subcategory of the category ${ }^{*} \operatorname{Mod}(R)$ of $\mathbb{Z}^{n}$-graded $R$-modules which has as objects the straight modules will be denoted Str. Let $\mathbf{1}=(1, \ldots, 1) \in \mathbb{Z}^{n}$. The shifted local cohomology modules $H_{I}^{r}(R)(\mathbf{- 1})$ supported on monomial ideals $I \subseteq R$ are straight modules. In order to avoid shiftings, we will consider instead the following (equivalent) category: 
Definition 3.3. We will say that a graded module $M$ is $\varepsilon$-straight if $M(\mathbf{- 1})$ is straight in the above sense. We denote $\varepsilon$-Str the full subcategory of ${ }^{*} \operatorname{Mod}(R)$ which has as objects the $\varepsilon$-straight modules.

If $M$ is a $A_{n}(\mathbb{C})$-module, then $\mathcal{M}^{a n}:=\mathcal{O}_{X} \otimes_{R} M$ has a natural $\mathcal{D}_{X}$-module structure. This allows to define a functor

$$
\begin{aligned}
(-)^{a n}: \operatorname{Mod}\left(A_{n}(\mathbb{C})\right) & \longrightarrow \operatorname{Mod}\left(\mathcal{D}_{X}\right) \\
M & \longrightarrow \mathcal{M}^{a n} \\
f & \longrightarrow i d \otimes f
\end{aligned}
$$

On the other hand, any $\varepsilon$-straight module $M$ can be endowed with a functorial $A_{n}(\mathbb{C})$-module structure extending its $R$-module structure, see [13]. Then, we have:

Theorem 3.4. ([2, 4.3]) The functor

$$
(-)^{a n}: \varepsilon-\mathbf{S t r} \longrightarrow \mathcal{D}_{v=0}^{T}
$$

is an equivalence of categories.

The following lemma (which in particular gives the fully faithfulness of $(-)^{a n}$ ) will be useful in the sequel.

Lemma 3.5. ([2, 4.4]) Let $M, N$ be $\varepsilon$-straight modules. For all $i \geq 0$, we have functorial isomorphisms

$$
{ }^{*} \operatorname{Ext}_{R}^{i}(M, N) \cong \operatorname{Ext}_{\mathcal{D}_{v=0}^{T}}^{i}\left(\mathcal{M}^{a n}, \mathcal{N}^{a n}\right)
$$

\section{The graded structure of $\mathcal{D}_{X}$-modules with variation zero}

Let $\mathcal{M} \in \mathcal{D}_{v=0}^{T}$ be a regular holonomic $\mathcal{D}_{X}$-module with variation zero and let $M \in \varepsilon-\mathbf{S t r}$ be the corresponding $\varepsilon$-straight module. Our aim in this section is to describe the $n$-hypercube corresponding to $\mathcal{M}$ from the $\varepsilon$-straight module structure of $M$.

Note that a Nilsson class function

$$
f=\sum_{\beta, m} \varphi_{\beta, m}(\mathbf{x})(\log \mathbf{x})^{m} \mathbf{x}^{\beta}
$$

is a solution of a module with variation zero if and only if $m=\mathbf{0}=(0, \ldots, 0) \in \mathbb{Z}^{n}$ and $\alpha \in \mathbb{Z}^{n}$, i.e. $f \in \mathcal{O}_{X}\left[\frac{1}{x_{1} \cdots x_{n}}\right]$. So, in order to determine the vertices of 
the $n$-hypercube for any module $\mathcal{M} \in \mathcal{D}_{v=0}^{T}$, we only have to consider the following spaces of solutions:

$$
\mathcal{E}_{\alpha}:=\frac{\mathcal{O}_{X}\left[\frac{1}{x_{1} \cdots x_{n}}\right]}{\sum_{\alpha_{k}=1} \mathcal{O}_{X}\left[\frac{1}{x_{1} \cdots \widehat{x_{k}} \cdots x_{n}}\right]} \subseteq \frac{\Gamma_{\prod_{i=1}^{n} V_{i}} \mathcal{O}_{X}}{\sum_{\alpha_{k}=1} \Gamma_{\mathbb{C}_{k} \times \prod_{i \neq k} V_{i} \mathcal{O}_{X}}}=\mathcal{S}_{\alpha}, \quad \alpha \in\{0,1\}^{n}
$$

In particular, we can give another description of the vertices of the $n$-hypercube of a module with variation zero. They are the vector spaces

$$
\mathcal{M}_{\alpha}:=\operatorname{Hom}_{\mathcal{D}_{X, 0}}\left(\mathcal{M}_{0}, \mathcal{E}_{\alpha, 0}\right) \text {. }
$$

This description makes the vertices of the $n$-hypercube more treatable due to the fact that $\mathcal{E}_{\alpha}$ are also modules with variation zero. In the sequel we will denote for simplicity $E_{\alpha}$ the corresponding $\varepsilon$-straight module. It is easy to see that

$$
E_{\alpha}={ }^{*} \mathrm{E}_{R}\left(R / \mathfrak{p}_{\alpha}\right)(\mathbf{1}) \cong \frac{R\left[\frac{1}{x_{1} \cdots x_{n}}\right]}{\sum_{\alpha_{k}=1} R\left[\frac{1}{x_{1} \cdots \widehat{x_{k}} \cdots x_{n}}\right]}
$$

where if $\alpha=\left(\alpha_{1}, \ldots, \alpha_{n}\right)$ and $\mathfrak{p}_{\alpha}$ denotes the monomial prime ideal $\left(x_{1}^{\alpha_{1}}, \ldots, x_{n}^{\alpha_{n}}\right)$, ${ }^{*} \mathrm{E}_{R}\left(R / \mathfrak{p}_{\alpha}\right)$ is the graded injective envelope of $R / \mathfrak{p}_{\alpha}$.

REMARK 4.1. The above facts mean, roughly speaking, that for a module of variation zero its solutions are algebraic. This indicates how to define the category of the modules of variation zero in the algebraic context. Namely, let $k$ be any field of characteristic zero and $R=k\left[x_{1}, \ldots, x_{n}\right]$. Then, the category of algebraic $D$ modules with variation zero is the category of straight $R$-modules as a full abelian subcategory of the category of $A_{n}(k)$-modules (the algebraic $D$-modules). One has to point out that this category is not closed under extensions as a subcategory of the category of algebraic $D$-modules. It is also clear that one can define over $k$ the category of $n$-hypercubes whose variation maps are zero. Then, by flat base change, one can see that by taking algebraic solutions, that is, the $k$-vector spaces $M_{\alpha}:=$ $\operatorname{Hom}_{A_{n}(k)}\left(M, E_{\alpha}\right)$, one obtains an equivalence between the category of algebraic $D$ modules with variation zero and the category of $n$-hypercubes over $k$ with variation zero. Similarly, by flat base change, one could also obtain a similar equivalence in the case of the formal power series ring $k\left[\left[x_{1}, \ldots, x_{n}\right]\right]$. All the results that come in the sequel could be then formulated in these more general settings, but for simplicity we shall only consider the analytic case.

We have to point out that, by using $[\mathbf{2}, 3.1]$, the vertices $\mathcal{M}_{\alpha}$ of the $n$-hypercube corresponding to $\mathcal{M}$ are isomorphic to the graded pieces $M_{-\alpha}$ of the corresponding $\varepsilon$-straight module $M$ for all $\alpha \in\{0,1\}^{n}$. But, in order to reflect the $\varepsilon$-straight module structure of $M$, we will consider the following description, which is the main result of this section. 
TheOREM 4.2. Let $\mathcal{M} \in \mathcal{D}_{v=0}^{T}$ be a regular holonomic $\mathcal{D}_{X}$-module with variation zero and $M \in \varepsilon-\mathbf{S t r}$ be the corresponding $\varepsilon$-straight module. Denote by $\left(M_{-\alpha}\right)^{*}$ the dual of the $\mathbb{C}$-vector space defined by the piece of $M$ of multidegree $-\alpha$, for all $\alpha \in\{0,1\}^{n}$. Then, there are isomorphisms

$$
\mathcal{M}_{\alpha} \cong\left(M_{-\alpha}\right)^{*}
$$

such that the following diagram commutes:

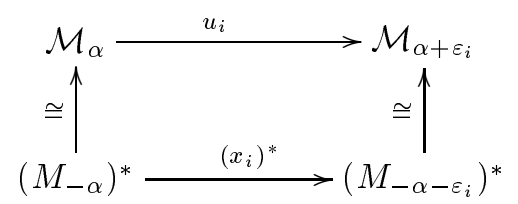

where $\left(x_{i}\right)^{*}$ is the dual of the multiplication by $x_{i}$.

Proof. By using the isomorphism given in Lemma 3.5, we only have to describe the $\mathbb{C}$-vector space ${ }^{*} \operatorname{Hom}_{R}\left(M, E_{\alpha}\right)$ in order to prove the existence of the isomorphisms.

Any map $f \in{ }^{*} \operatorname{Hom}_{R}\left(M, E_{\alpha}\right)$ is determined by the pieces $f_{-\beta}, \beta \in\{0,1\}^{n}$ due to the fact that $M$ and $E_{\alpha}$ are $\varepsilon$-straight modules. Notice that $f_{-\beta}=0$, for all $\beta<\alpha$, due to the fact that $\mathfrak{p}_{\alpha}$ is the unique associated prime of $E_{\alpha}$. On the other side, for all $i$ such that $\alpha_{i}=0$ we have

$$
f_{-\alpha-\varepsilon_{i}}: M_{-\alpha-\varepsilon_{i}} \stackrel{x_{i}}{\longrightarrow} M_{-\alpha} \stackrel{f_{-\alpha}}{\longrightarrow}\left[E_{\alpha}\right]_{-\alpha} \stackrel{\frac{1}{x_{i}}}{\longrightarrow}\left[E_{\alpha}\right]_{-\alpha-\varepsilon_{i}}
$$

i.e. $f_{-\alpha-\varepsilon_{i}}:=\frac{1}{x_{i}} \circ f_{-\alpha} \circ x_{i}$. By using this construction in an analogous way we can describe $f_{-\beta}$ for all $\beta \geq \alpha$. In particular, the map $f \in{ }^{*} \operatorname{Hom}_{R}\left(M, E_{\alpha}\right)$ is determined by the piece $f_{-\alpha}$. Namely, we have the isomorphism:

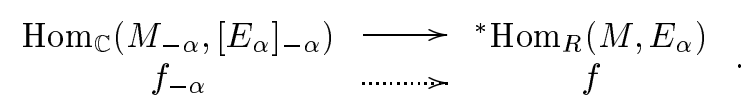

Finally, since $\left[E_{\alpha}\right]_{-\alpha}$ is the $\mathbb{C}$-vector space spanned by $\frac{1}{\mathbf{x}^{\alpha}}$, the multiplication by $\mathbf{x}^{\alpha}$ gives an isomorphism:

$$
\begin{array}{ccc}
\operatorname{Hom}_{\mathbb{C}}\left(M_{-\alpha},\left[E_{\alpha}\right]_{-\alpha}\right) & \longrightarrow & \operatorname{Hom}_{\mathbb{C}}\left(M_{-\alpha}, \mathbb{C}\right)=\left(M_{-\alpha}\right)^{*} \\
f_{-\alpha} & \cdots \cdots \ldots .> & \mathbf{x}^{\alpha} f_{-\alpha}
\end{array}
$$

where we consider $\mathbb{C}$ as the $\mathbb{C}$-vector space spanned by 1 .

Once the vertices of the $n$-hypercube are determined, we only have to describe the linear map

$$
u_{i}:{ }^{*} \operatorname{Hom}_{R}\left(M, E_{\alpha}\right) \longrightarrow{ }^{*} \operatorname{Hom}_{R}\left(M, E_{\alpha+\varepsilon_{i}}\right)
$$

induced by the natural quotient maps $E_{\alpha} \longrightarrow E_{\alpha+\varepsilon_{i}}$.

Let $f \in{ }^{*} \operatorname{Hom}_{R}\left(M, E_{\alpha}\right)$ be a morphism described by the linear map $f_{-\alpha} \in$ $\operatorname{Hom}_{\mathbb{C}}\left(M_{-\alpha},\left[E_{\alpha}\right]_{-\alpha}\right)$. Then, the corresponding morphism $\bar{f} \in{ }^{*} \operatorname{Hom}_{R}\left(M, E_{\alpha+\varepsilon_{i}}\right)$ induced by the quotient map $E_{\alpha} \rightarrow E_{\alpha+\varepsilon_{i}}$ is described by the linear map $f_{-\alpha-\varepsilon_{i}} \in$ 
$\operatorname{Hom}_{\mathbb{C}}\left(M_{-\alpha-\varepsilon_{i}},\left[E_{\alpha+\varepsilon_{i}}\right]_{-\alpha-\varepsilon_{i}}\right)$ defined as:

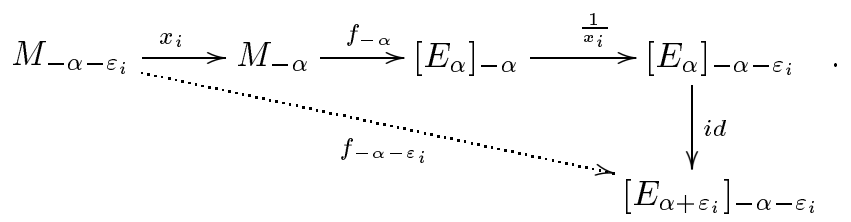

In particular we have the commutative diagram:

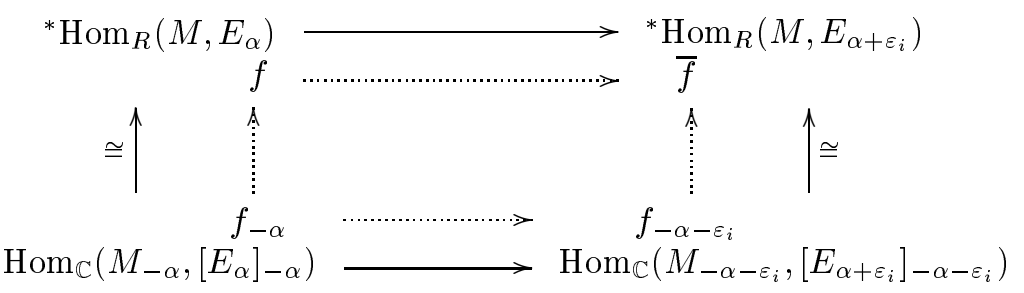

Then we are done because the following diagram is commutative:

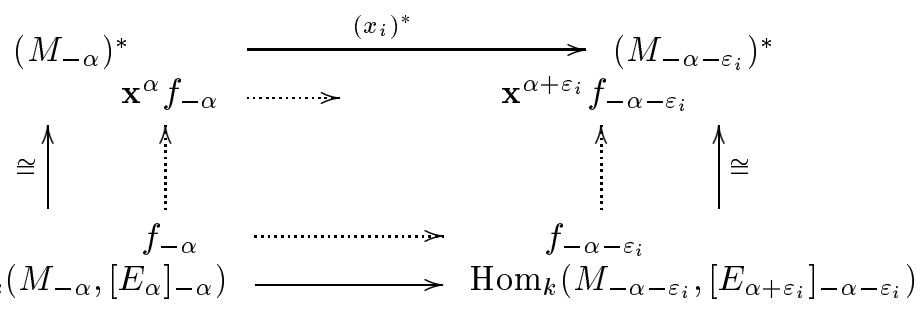

Namely, we have:

$$
\left(x_{i}\right)^{*}\left(\mathbf{x}^{\alpha} f_{-\alpha}\right)=\mathbf{x}^{\alpha} f_{-\alpha} x_{i}=\mathbf{x}^{\alpha+\varepsilon_{i}} \frac{1}{x_{i}} f_{-\alpha} x_{i}=\mathbf{x}^{\alpha+\varepsilon_{i}} f_{-\alpha-\varepsilon_{i}} .
$$

\section{Local cohomology modules: Topological interpretation}

Let $I=\left(\mathbf{x}^{\alpha_{1}}, \ldots, \mathbf{x}^{\alpha_{s}}\right) \subseteq R, \alpha_{i} \in\{0,1\}^{n}$, be a minimal system of generators of a squarefree monomial ideal $I$. Consider the Cech complex

$$
\check{C}_{I}^{\bullet}: \quad 0 \longrightarrow R \stackrel{d_{0}}{\longrightarrow} \bigoplus_{1 \leq i_{1} \leq s} R\left[\frac{1}{\mathbf{x}^{\alpha_{\mathbf{i}_{1}}}}\right] \stackrel{d_{1}}{\longrightarrow} \cdots \stackrel{d_{s-1}}{\longrightarrow} R\left[\frac{1}{\mathbf{x}^{\alpha_{1}} \cdots \mathbf{x}^{\alpha_{\mathbf{s}}}}\right] \longrightarrow 0 .
$$

By using the equivalence of categories given in Section 3 and the contravariance of the functor $\operatorname{Mod}\left(\mathcal{D}_{X}\right)_{h r}^{T} \longrightarrow \mathcal{C}_{n}$, the $n$-hypercube corresponding to a local cohomology module $H_{I}^{r}(R)$ supported on $I$ may be described as follows:

- Vertices: $\left(\mathcal{H}_{I}^{r}(\mathcal{R})\right)_{\alpha}=H_{r}\left({ }^{*} \operatorname{Hom}_{R}\left(\check{C}_{I}^{\bullet}, E_{\alpha}\right)\right)$.

- Linear maps: $u_{i}: H_{r}\left({ }^{*} \operatorname{Hom}_{R}\left(\check{C}_{I}^{\bullet}, E_{\alpha}\right)\right) \longrightarrow H_{r}\left({ }^{*} \operatorname{Hom}_{R}\left(\check{C}_{I}^{\bullet}, E_{\alpha+\varepsilon_{i}}\right)\right)$, induced by the quotient map $E_{\alpha} \longrightarrow E_{\alpha+\varepsilon_{i}}$.

To illustrate this computations we present the following: 
Example 5.1. Let $R=\mathbb{C}\left[x_{1}, x_{2}, x_{3}\right]$. Consider the ideal:

- $I=\left(x_{1} x_{2}, x_{1} x_{3}, x_{2} x_{3}\right)$.

Applying [1, 3.8] there is a local cohomology module different from zero and its characteristic cycle is

$$
C C\left(H_{I}^{2}(R)\right)=T_{X_{(1,1,0)}}^{*} X+T_{X_{(1,0,1)}}^{*} X+T_{X_{(0,1,1)}}^{*} X+2 T_{X_{(1,1,1)}}^{*} X .
$$

In particular, we only have to study the vertices of the $n$-hypercube $\left(\mathcal{H}_{I}^{2}(\mathcal{R})\right)_{\alpha}$ for $\alpha=(1,1,0),(1,0,1),(0,1,1),(1,1,1)$.

We have the Čech complex:

$$
\begin{aligned}
& R\left[\frac{1}{x_{1} x_{2}}\right] R\left[\frac{1}{x_{1} x_{2} x_{3}}\right] \\
& \check{C}_{I}^{\bullet:} 0 \longrightarrow R \stackrel{\oplus}{\longrightarrow} R\left[\frac{1}{x_{1} x_{3}}\right] \stackrel{d_{0}}{\longrightarrow} R\left[\frac{1}{x_{1} x_{2} x_{3}}\right] \stackrel{d_{1}}{\longrightarrow} R\left[\frac{1}{x_{1} x_{2} x_{3}}\right] \longrightarrow 0 \\
& R\left[\frac{1}{x_{2} x_{3}}\right] \quad R\left[\frac{1}{x_{1} x_{2} x_{3}}\right]
\end{aligned}
$$

- The complex ${ }^{*} \operatorname{Hom}_{R}\left(\check{C}_{I}^{\bullet}, E_{(1,1,1)}\right)$ is of the form:

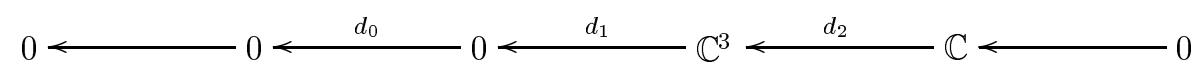

where $d_{2}=\left(\begin{array}{r}1 \\ -1 \\ 1\end{array}\right)$. Then, we get the vertex:

$$
\left(\mathcal{H}_{I}^{2}(\mathcal{R})\right)_{(1,1,1)}=H_{2}\left({ }^{*} \operatorname{Hom}_{R}\left(\check{C}_{I}^{\bullet}, E_{(1,1,1)}\right)\right)=\mathbb{C}^{2} .
$$

- The complexes ${ }^{*} \operatorname{Hom}_{R}\left(\check{C}_{I}^{\bullet}, E_{\alpha}\right)$, for $\alpha=(1,1,0),(1,0,1),(0,1,1)$, are of the form:

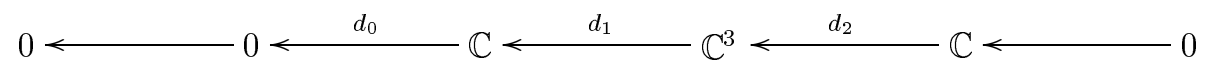

where $d_{1}=\left(\begin{array}{lll}1 & 0 & -1\end{array}\right)$ and $d_{2}=\left(\begin{array}{r}1 \\ -1 \\ 1\end{array}\right)$. Then, we get the vertices:

$$
\left(\mathcal{H}_{I}^{2}(\mathcal{R})\right)_{\alpha}=H_{2}\left({ }^{*} \operatorname{Hom}_{R}\left(\check{C}_{I}^{\bullet}, E_{\alpha}\right)\right)=\mathbb{C}, \text { for } \alpha=(1,1,0),(1,0,1),(0,1,1) .
$$


Computing the linear maps among these vertices in an adequate basis we get the $n$-hypercube:

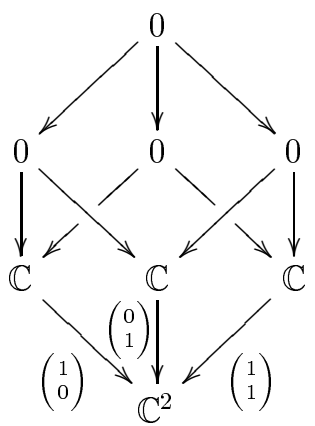

The $n$-hypercubes corresponding to the local cohomology modules supported on squarefree monomial ideals can be better described in topological terms by using the cellular structure of the complexes used in their computation.

As before, let $I=\left(\mathbf{x}^{\alpha_{1}}, \ldots, \mathbf{x}^{\alpha_{s}}\right) \subseteq R$ be a minimal system of generators of a squarefree monomial ideal $I$. Consider the Čech complex:

$$
\check{C}_{I}^{\bullet}: 0 \longrightarrow R \stackrel{d_{0}}{\longrightarrow} \bigoplus_{1 \leq i_{1} \leq s} R\left[\frac{1}{\mathbf{x}^{\alpha_{\mathbf{i}_{1}}}}\right] \stackrel{d_{1}}{\longrightarrow} \cdots \stackrel{d_{s-1}}{\longrightarrow} R\left[\frac{1}{\mathbf{x}^{\alpha_{1}} \cdots \mathbf{x}^{\alpha_{\mathbf{s}}}}\right] \longrightarrow 0 .
$$

Vertices: To describe the vertices of the $n$-hypercubes corresponding to the local cohomology modules $H_{I}^{r}(R)$ we only have to notice the following:

- Applying the functor ${ }^{*} \operatorname{Hom}_{R}\left(-, E_{\alpha}\right)$ to the Čech complex $\breve{C}_{I}^{\bullet}$ in the case $\alpha=\mathbf{0}=(0, \ldots, 0) \in\{0,1\}^{n}$, we obtain the complex:

$$
{ }^{*} \operatorname{Hom}_{R}\left(\check{C}_{I}^{\bullet}, E_{\mathbf{0}}\right): 0 \longleftarrow \mathbb{C} \stackrel{d_{0}}{\longleftarrow} \mathbb{C}^{s} \stackrel{d_{1}}{\longleftarrow} \cdots \stackrel{d_{s-1}}{\longleftarrow} \mathbb{C} \longleftarrow 0 .
$$

This complex may be identified with the augmented relative simplicial chain complex $\widetilde{\mathcal{C}}_{\bullet}(\Delta ; \mathbb{C})$, where $\Delta$ is the full simplicial complex whose vertices $\left\{x_{1}, \ldots, x_{s}\right\}$ are labelled by the minimal system of generators of $I$.

- In general, for any $\alpha \in\{0,1\}^{n}$, the terms of the complex ${ }^{*} \operatorname{Hom}_{R}\left(\check{C}_{I}^{\bullet}, E_{\alpha}\right)$ are:

$$
{ }^{*} \operatorname{Hom}_{R}\left(R\left[\frac{1}{\mathbf{x}^{\beta}}\right], E_{\alpha}\right)= \begin{cases}\mathbb{C} & \text { if } \beta \geq \alpha \\ 0 & \text { otherwise }\end{cases}
$$

Namely, from the augmented relative simplicial chain complex $\widetilde{\mathcal{C}}_{\bullet}(\Delta ; \mathbb{C})$, we are taking out the pieces corresponding to the faces

$$
\sigma_{1-\beta}:=\left\{x_{1}, \ldots, x_{s}\right\} \backslash\left\{x_{i} \mid \beta_{i}=1\right\} \in \Delta \text { such that } \beta \nsupseteq \alpha .
$$

Let $T_{\alpha}:=\left\{\sigma_{1-\beta} \in \Delta \mid \beta \nsupseteq \alpha\right\}$ be a simplicial subcomplex of $\Delta$. Then, the complex ${ }^{*} \operatorname{Hom}_{R}\left(\breve{C}_{I}^{\bullet}, E_{\alpha}\right)$ may be identified with the augmented relative simplicial chain complex $\widetilde{\mathcal{C}}_{\bullet}\left(\Delta, T_{\alpha} ; \mathbb{C}\right)$ associated to the pair $\left(\Delta, T_{\alpha}\right)$. By taking homology, 
the vertices of the $n$-hypercubes corresponding to the local cohomology modules $H_{I}^{r}(R)$ are:

$$
\left(\mathcal{H}_{I}^{r}(\mathcal{R})\right)_{\alpha}=H_{r}\left({ }^{*} \operatorname{Hom}_{R}\left(\check{C}_{I}^{\bullet}, E_{\alpha}\right)\right)=\widetilde{H}_{r-1}\left(\Delta, T_{\alpha} ; \mathbb{C}\right)=\widetilde{H}_{r-2}\left(T_{\alpha} ; \mathbb{C}\right)
$$

where the last assertion comes from the fact that $\Delta$ is contractible.

Linear maps: By using the description of the vertices we notice the following:

- The morphism of complexes $\left.{ }^{*} \operatorname{Hom}_{R}\left(\check{C}_{I}^{\bullet}, E_{\alpha}\right)\right) \longrightarrow{ }^{*} \operatorname{Hom}_{R}\left(\check{C}_{I}^{\bullet}, E_{\alpha+\varepsilon_{i}}\right)$, induced by the quotient map $E_{\alpha} \longrightarrow E_{\alpha+\varepsilon_{i}}$ is nothing but the morphism of complexes $\widetilde{\mathcal{C}} \cdot\left(\Delta, T_{\alpha} ; \mathbb{C}\right) \longrightarrow \widetilde{\mathcal{C}} \bullet\left(\Delta, T_{\alpha+\varepsilon_{i}} ; \mathbb{C}\right)$ induced by the inclusion $T_{\alpha} \subseteq T_{\alpha+\varepsilon_{i}}$. By taking homology, the linear maps $u_{i}$ of the $n$-hypercubes corresponding to the local cohomology modules $H_{I}^{r}(R)$ are:

$$
\nu_{i}: \widetilde{H}_{r-2}\left(T_{\alpha} ; \mathbb{C}\right) \longrightarrow \widetilde{H}_{r-2}\left(T_{\alpha+\varepsilon_{i}} ; \mathbb{C}\right)
$$

induced by the inclusion $T_{\alpha} \subseteq T_{\alpha+\varepsilon_{i}}$.

Collecting the previous results we get:

Proposition 5.2. The n-hypercubes corresponding to local cohomology modules $H_{I}^{r}(R)$ supported on squarefree monomial ideals $I \subseteq R$ are:

- Vertices: $\left(\mathcal{H}_{I}^{r}(\mathcal{R})\right)_{\alpha} \cong \widetilde{H}_{r-2}\left(T_{\alpha} ; \mathbb{C}\right)$.

- Linear maps: We have the following commutative diagram:

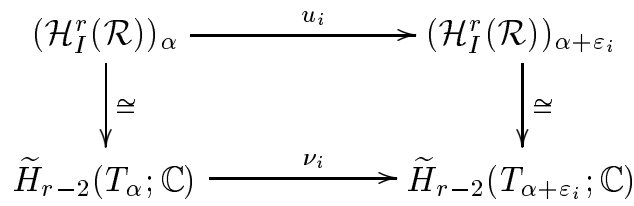

where $\nu_{i}$ is induced by the inclusion $T_{\alpha} \subseteq T_{\alpha+\varepsilon_{i}}$.

Remark 5.3. By using the previous result and Theorem 4.2 we recover the result on the module structure of the local cohomology modules $H_{I}^{r}(R)$ given by M. Mustaţă [10]. Namely, if $(-)^{*}$ denotes the dual of a $\mathbb{C}$-vector space, the graded pieces of $H_{I}^{r}(R)$ are:

$$
\left[H_{I}^{r}(R)\right]_{-\alpha} \cong\left(\widetilde{H}_{r-2}\left(T_{\alpha} ; \mathbb{C}\right)\right)^{*} \cong \widetilde{H}^{r-2}\left(T_{\alpha} ; \mathbb{C}\right), \quad \alpha \in\{0,1\}^{n}
$$


and the multiplication map $x_{i}:\left[H_{I}^{r}(R)\right]_{-\alpha-\varepsilon_{i}} \longrightarrow\left[H_{I}^{r}(R)\right]_{-\alpha}$ is determined by the following commutative diagram:

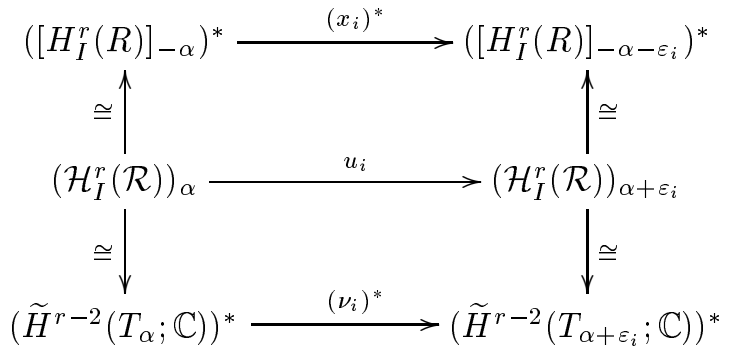

where $\nu_{i}$ is induced by the inclusion $T_{\alpha} \subseteq T_{\alpha+\varepsilon_{i}}$.

\section{References}

[1] J. Àlvarez Montaner, Characteristic cycles of local cohomology modules of monomial ideals, J. Pure Appl. Algebra, 150 (2000), 1-25.

[2] J. Àlvarez Montaner, R. García López and S. Zarzuela, Local cohomology, arrangements of subspaces and monomial ideals, to appear in Adv. in Math.

[3] J.- E. Björk, Rings of differential operators, North Holland Mathematics Library, 1979.

[4] J.- E. Björk, Analytic D-modules and applications, Mathematics and its Applications, Vol. 247, Kluwer Academic Publishers, Dordrecht, 1993.

[5] A. Borel et. al, Algebraic D-modules, Perspectives in Mathematics, Vol. 2, Academic Press, New York, 1987.

[6] A. Galligo, M. Granger and Ph. Maisonobe, D-modules et faisceaux pervers dont le support singulier est un croisement normal, Ann. Inst. Fourier, 35 (1985), 1-48.

[7] A. Galligo, M. Granger and Ph. Maisonobe, $\mathcal{D}$-modules et faisceaux pervers dont le support singulier est un croisement normal. II, in Differential systems and singularities (Luminy, 1983), Astérisque, 130 (1985), 240-259.

[8] S. Goto and K. Watanabe, On Graded Rings, II ( $\mathbb{Z}^{n}$ - graded rings), Tokyo J. Math., 1-2 (1978), 237-261.

[9] G. Lyubeznik, Finiteness properties of local cohomology modules (an application of $\mathcal{D}$ modules to commutative algebra), Invent. Math., 113 (1993), 41-55.

[10] M. Mustaţă, Local Cohomology at Monomial Ideals, J. Symbolic Comput., 29 (2000), 709720 .

[11] U. Walther, Algorithmic computation of local cohomology modules and the cohomological dimension of algebraic varieties, J. Pure Appl. Algebra, 139 (1999), 303-321.

[12] U. Walther, On the Lyubeznik numbers of a local ring, Proc. Amer. Math. Soc., 129-6 (2001), 1631-1634.

[13] K. Yanagawa, Bass numbers of local cohomology modules with supports in monomial ideals, Math. Proc. Cambridge Philos. Soc., 131 (2001), 303-321.

Departament de Matemàtica Aplicada I, Universitat Politècnica de Catalunya, Avinguda Diagonal 647, E-08028 Barcelona, SPAIN

E-mail address: Josep.Alvarez@upc.es

Departament d’Àlgebra i Geometria, Universitat de Barcelona, Gran Via de les Corts Catalanes 585, E-08007 Barcelona, SPAiN

E-mail address: zarzuela@mat.ub.es 